7. Block C, Robenshtok E, Simhon A, Shapiro M. Evaluation of chlorhexidine and povidone iodine activity against methicillin-resistant Staphylococcus aureus and vancomycin-resistant Enterococcus faecalis using a surface test. $J$ Hosp Infect 2000;46:147-152.

8. Shenoy ES, Noubary F, Kim JY, et al. Concordance of PCR and culture from nasal swabs for detection of methicillin-resistant Staphylococcus aureus in a setting of concurrent antistaphylococcal antibiotics. J Clin Microbiol 2014;52:1235-1237.
9. Rezapoor M, Nicholson T, Tabatabaee RM, Chen AF, Maltenfort MG, Parvizi J. Povidone-iodine-based solutions for decolonization of nasal Staphylococcus aureus: a randomized, prospective, placebo-controlled study. J Arthroplasty 2017;32:2815-2819.

10. Anderson MJ, David ML, Scholz M, et al. Efficacy of skin and nasal povidone-iodine preparation against mupirocin-resistant methicillinresistant Staphylococcus aureus and S. aureus within the anterior nares. Antimicrob Agents Chemother 2015;59:2765-2773.

\title{
Whole-genome sequence analyses by a new easy-to-use software solution support the suspicion of a neonatal ward outbreak of methicillin-resistant Staphylococcus aureus (MRSA) and transmission between hospitals
}

\author{
Marie Louise Slott Jensen ${ }^{1,2,3}$, Ming Chen ${ }^{3,4}$, Mette Detlefsen², Janne Kudsk Klitgaard ${ }^{2,6}$, Thomas Emil Andersen ${ }^{1,2}$ and \\ Michael Kemp ${ }^{3,5,7}$ (1) \\ ${ }^{1}$ Department of Clinical Research, University of Southern Denmark, Odense, Denmark, ${ }^{2}$ Department of Clinical Microbiology, Odense University Hospital, Odense, \\ Denmark, ${ }^{3}$ Center for Bacterial Genomics in Southern Denmark, University of Southern Denmark, Odense, Denmark, ${ }^{4}$ Department of Clinical Microbiology, \\ Hospital Southern Jutland, Soenderborg, Denmark, ${ }^{5}$ Department of Regional Health Research, University of Southern Denmark, Odense, Denmark, ${ }^{6}$ Department \\ Biochemistry and Molecular Biology, Research unit of Molecular Microbiology, University of Southern Denmark, Odense, Denmark and ${ }^{7}$ Department of Clinical \\ Microbiology, Zealand University Hospital, Slagelse, Denmark
}

To the Editor-Methicillin-resistant Staphylococcus aureus (MRSA) occasionally causes outbreaks in hospitals, often in departments where newborns receive treatment. ${ }^{1,2}$ Hospital-acquired infections by MRSA may have a lethal outcome ${ }^{3}$ and expenses for infection control measures for containing hospital outbreaks may become very high. ${ }^{4}$ Surveillance of MRSA is essential for early detection and interruption of transmission. Whole-genome sequencing (WGS) is successfully used for comparing isolates of MRSA., ${ }^{1,2,5}$ Multilocus sequence typing (MLST) is a traditional high-resolution typing method based on allele sequences. MLST sequence types (STs) can be extracted from WGS data. An even higher resolution is obtained when the number of alleles analyzed is increased as in the WGS-based method core genome MLST. ${ }^{6}$ Both methods have shown their value in several studies of MRSA. ${ }^{2,4,7} 1928$ Diagnostics is a WGS-based online solution that requires no bioinformatic experience from the user. The system allows comparison and characterization of several bacteria, including S. aureus (9). Raw sequence data are uploaded to the 1928 Diagnostics server (https://www.1928diagnostics.com). A builtin quality check ensures that sequences of poor quality (low sequencing coverage) are not processed. For S. aureus, results include individual MLST ST, antibiotic resistance mechanisms (resistance genes and mutations associated with resistance), and virulence gene profiles. A core genome sequence-based cluster tree shows the relatedness of isolates. Published experience on the use of 1928 Diagnostics for hospital surveillance is limited. We tested 1928 Diagnostics in a retrospective analysis of a neonatal ward

Author for correspondence: Michael Kemp, E-mail: mkemp@regionsjaelland.dk.

Cite this article: Slott Jensen ML, et al. (2022). Whole-genome sequence analyses by a new easy-to-use software solution support the suspicion of a neonatal ward outbreak of methicillin-resistant Staphylococcus aureus (MRSA) and transmission between hospitals. Infection Control \& Hospital Epidemiology, 43: 947-949, https://doi.org/ $10.1017 /$ ice.2021.123 outbreak of MRSA ST22 after transfer of a patient from another hospital.

The index patient was a newborn transferred to hospital A from the neighboring hospital B on May 3, 2014. On June 1, MRSA was cultured from blood and a skin abscess from the patient. The isolate was typed as ST22 at Statens Serum Institut, Copenhagen, as part of the national surveillance of MRSA. MRSA ST22 was isolated from a diagnostic sample from another patient at the ward on June 4 , and patients and staff members were screened for MRSA. MRSA ST22 was isolated from additional 6 individuals, including 1 mother and 2 staff members. Infection control interventions included, among other measures, an audit in infection control at the ward focused on reinforcing standard precaution measures, replacement of worn utensils and equipment, and thorough cleaning and disinfection of parts of the ward. No MRSA ST22 were isolated from patients, relatives, or staff members after June 16. On July 12, MRSA ST22 was cultured from a patient, previously admitted to the affected ward concomitant with the index patient. No further transmission was observed.

We included isolates of MRSA ST22 (1) from 9 individuals suspected of being infected during the outbreak, (2) from 9 individuals from the same area collected over the preceding 7 years, and (3) from 27 individuals from the neighboring hospital area in the same year as the suspected outbreak. One isolate was analyzed from each individual. WGS was performed on a Miseq (Illumina, San Diego, CA). ${ }^{4}$

The genome sequences confirmed all isolates as MRSA ST22 carrying the resistance genes bla $Z$ and mecA. One isolate was typed as a recombinant sccmec type, while all other isolates were sccmec type IV. Genes encoding Panton-Valentine leucocidin, toxic shock syndrome toxin, and exfoliative toxins were not detected. All isolates had 1 or more mutations associated with resistance to ciprofloxacin (Fig. 1). No other genetic markers of resistance to antibiotics were detected in the outbreak isolates. The 


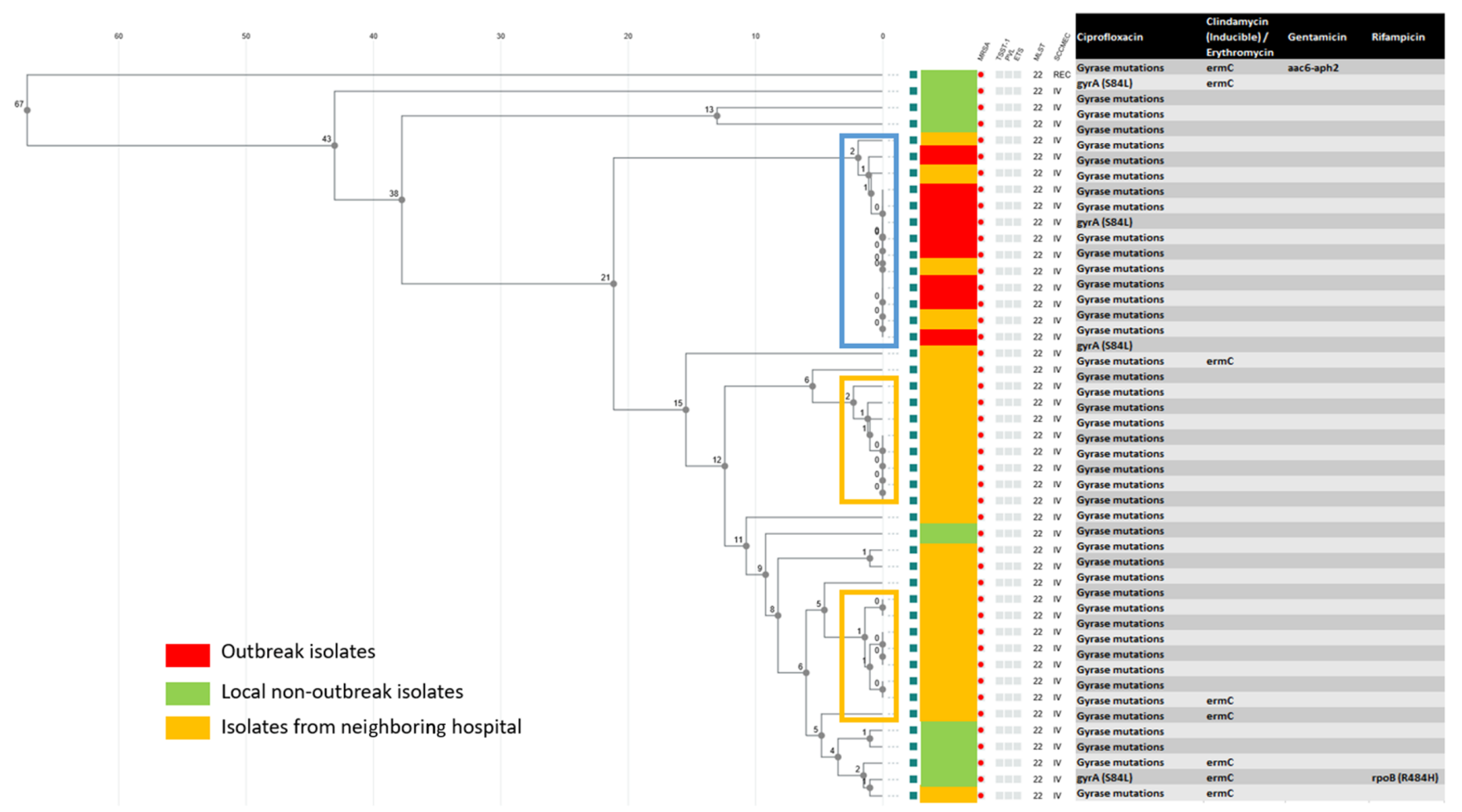

Fig. 1. Core genome based phylogenetic tree showing individual MRSA ST22 isolates. The 9 outbreak isolates (red) clearly clustered with 4 of the isolates from the neighboring area (yellow), while nonoutbreak isolates from the same area (green) and other isolates from the neighboring area were more distant. In addition to the cluster of outbreak isolates from hospital A and isolates from hospital B, marked by a blue rectangle, 2 clusters of isolates with highly similar core genomes, marked by yellow rectangles, were detected. Numbers at the nodes indicate average allelic distances between samples and clusters of samples in the tree. Genes and mutations associated with antibiotic resistance are shown for individual isolates. "Gyrase mutations" indicates that $>1$ mutation associated with resistance to ciprofloxacin was detected.

erythromycin/inducible clindamycin resistance gene ermC was detected in 8 nonoutbreak isolates. One isolate carried the highlevel gentamicin resistance gene aac6-aph2. A mutation associated with resistance to rifampicin was detected in one isolate. The study aimed at testing 1928 Diagnostics for local outbreak investigation and concordance between genetic markers of resistance and phenotypic resistance testing was not pursued. Previous studies have investigated genetic resistance determination by 1928 Diagnostics in methicillin-resistant S. aureus and S. argenteus. ${ }^{8-10}$

In the cluster analysis we arbitrarily defined a cluster as 5 or more isolates with an allelic distance of $<5$. All suspected outbreak isolates were within one cluster (Fig. 1), confirming the suspicion of local transmission. In addition, 4 isolates from hospital B were also in this cluster. Based on MLST typing, the outbreak was assumed to be caused by a strain transferred from hospital B by the index patient. However, all isolates from hospital B in the outbreak cluster were cultured later than the isolate from the index patient. Thus, the higher resolution obtained from cgMLST did support suspicion of transmission of the outbreak strain between the hospitals but did not confirm this taking place via transfer of the index patient from hospital B. Another 2 clusters were detected among isolates from hospital B (Fig. 1).

Rapid typing of selected bacteria is essential for effective surveillance and early detection of outbreaks in hospitals. The simple procedure for uploading data and viewing results makes 1928 Diagnostics attractive to infection control units without bioinformatic expertise. Inclusion of low-quality sequence data in the analyses may hamper the strength of conclusions, and the integrated quality control is important when the system is used by professionals without experience in bioinformatics. Our results suggest that 1928 Diagnostics may be used by local infection control units without bioinformatic expertise. However, the retrospective design is a limitation of this study, and prospective real-time studies are needed to establish the usefulness of 1928 Diagnostics and similar systems for surveillance.

\section{Acknowledgments.}

Financial support. The study was supported by grants for whole-genome sequencing from Odense University Hospital Research Fund and from Region Southern Denmark Research Fund (grant no. 16/41984).

Conflicts of interest. All authors report no conflicts of interest relevant to this article.

\section{References}

1. Harris SR, Cartwright EJ, Török ME, et al. Whole-genome sequencing for analysis of an outbreak of meticillin-resistant Staphylococcus aureus: a descriptive study. Lancet Infect Dis 2013;13:130-136.

2. Madigan T, Cunningham SA, Patel R, et al. Whole-genome sequencing for methicillin-resistant Staphylococcus aureus (MRSA) outbreak investigation in a neonatal intensive care unit. Infect Control Hosp Epidemiol 2018;39:1412-1418.

3. Nielsen RT, Kemp M, Holm A, et al. Fatal septicemia linked to transmission of MRSA clonal complex 398 in hospital and nursing home, Denmark. Emerg Infect Dis 2016;22:900-902.

4. Slott Jensen ML, Nielsine Skov M, Pries Kristiansen H, et al. Core genome multilocus sequence typing as an essential tool in a high-cost livestock-associated meticillin-resistant Staphylococcus aureus CC398 hospital outbreak. J Hosp Infect 2020;104:574-581. 
5. Bartels Bartels MD, Larner-Svensson H, Meiniche H, et al. Monitoring methicillin-resistant Staphylococcus aureus and its spread in Copenhagen, Denmark, 2013, through routine whole-genome sequencing. Euro Surveill 2015;20. doi: 10.2807/1560-7917.es2015.20.17.21112.

6. Uelze L, Grützke J, Borowiak M, Hammerl JA, Juraschek K, Deneke C, Tausch SH, Malorny B. Typing methods based on whole genome sequencing data. One Health Outlook 2020;2. doi.org/10.1186/s42522-020-0010-1.

7. Bartels MD, Worning P, Andersen LP, et al. Repeated introduction and spread of the MRSA clone t304/ST6 in northern Europe. Clin Microbiol Infect 2020. doi: 10.1016/j.cmi.2020.05.004.
8. Cunningham SA, Jeraldo PR, Schuetz AN, Heitman AA, Patel R. Staphylococcus aureus whole genome sequence-based susceptibility and resistance prediction using a clinically amenable workflow. Diagn Microbiol Infect Dis 2020 97:115060.

9. Enström J, Fröding I, Giske CG, et al. USA300 methicillin-resistant Staphylococcus aureus in Stockholm, Sweden, from 2008 to 2016. PLoS One 2018;13:e0205761.

10. Giske CG, Dyrkell F, Arnellos D, et al. Transmission events and antimicrobial susceptibilities of methicillin-resistant Staphylococcus argenteus in Stockholm. Clin Microbiol Infect 2019;25:1289.e5-1289.e8.

\title{
Neosartorya hiratsukae: Environmental isolation from intensive care units in an Italian hospital
}

\author{
Anna Prigitano $\mathrm{PhD}^{1}$ (1), Maria C. Esposto $\mathrm{BS}^{1}$, Davide Carnevali $\mathrm{MD}^{2}$, Emanuele Catena $\mathrm{MD}^{3}$, \\ Francesco Auxilia MD, $\mathrm{PROF}^{1,4}$, Silvana Castaldi MD, PROF ${ }^{1,5, a}$ and Luisa Romanò PhD, PROF ${ }^{1, a}$ \\ ${ }^{1}$ Department of Biomedical Sciences for Health, Università degli Studi di Milano, Milano, Italy, ${ }^{2}$ Postgraduate School in Public Health, Department of Biomedical \\ Sciences for Health, Università degli Studi di Milano, Milano, Italy, ${ }^{3}$ Department of Anaesthesiology and Intensive Care Unit, Luigi Sacco Hospital, Milano, Italy, \\ ${ }^{4}$ ASST Fatebenefratelli Sacco, Milano, Italy and ${ }^{5}$ Fondazione IRCCS Ca' Granda Ospedale Maggiore Policlinico di Milano, Italy
}

To the Editor-Fungi are opportunistic pathogens, ubiquitously distributed. Fungal colonization acquired from the environment might evolve into an invasive infection during hospitalization due to immunosuppressive treatments to which patients are exposed. Environmental surveillance in hospital settings requires special attention to prevent fungal and non-fungal infections. Invasive aspergillosis is one of the most important infections caused by Aspergillus. Neosartorya hiratsukae, teleomorph of the Aspergillus section Fumigati, is a rare fungus isolated for the first time in Japan from indoor air and from pasteurized aloe juice. ${ }^{1}$

Neosartorya hiratsukae is an opportunistic pathogen and only few cases of human infections have been reported worldwide. ${ }^{2-6}$ $N$. hiratsukae is often misidentified because it cannot be distinguished from A. fumigatus sensu stricto by conventional morphological macroscopic and microscopic analyses or by the extensively used matrix-assisted laser desorption/ionization time-of-flight mass spectrometry (MALDI-TOF-MS). However, rapid and accurate identification is important for therapeutic purposes due to the different patterns of susceptibility to antifungal drugs.

In this study, we investigated the environmental isolation of $N$. hiratsukae from 2 different intensive care units (ICUs) in a hospital in Milan, Italy. In January 2020, environmental sampling was conduted using contact plates as part of an environmental surveillance study. In total, 9 bed stations and 2 medical stations were sampled, for a total of 55 samples. After macroscopic and microscopic analyses, identification was confirmed by molecular analysis. Antifungal susceptibility testing was performed by broth microdilution assay according to European Committee on Antimicrobial Susceptibility Testing (EUCAST) to determine the minimum inhibitory concentration (MIC). ${ }^{7}$ The following

Author for correspondence: Dr Anna Prigitano, E-mail: anna.prigitano@unimi.it aAuthors of equal contribution.

Cite this article: Prigitano A, et al. (2022). Neosartorya hiratsukae: Environmental isolation from intensive care units in an Italian hospital. Infection Control \& Hospital Epidemiology, 43: 949-950, https://doi.org/10.1017/ice.2021.136 antifungals were tested: itraconazole, voriconazole, posaconazole, isavuconazole and amphotericin B (Sigma-Aldrich, St Louis, MO).

Fungi grew from $18.2 \%$ of the environmental samples; molds grew from 8 samples. We identified 2 mold colonies, isolated from a cooling fan and from the handles of a patient bed, as A. niger and A. fumigatus. In total, 13 colonies of filamentous fungi isolated from 6 samples showed slow growth, with restricted white colonies and light-brown reverse. In particular, in one ICU, 12 colonies grew from the scialytic lamps ( $n=7)$ of 2 different patient beds, from the computer keyboard of the medical station $(n=3)$, from the patient vital signs monitor $(\mathrm{n}=1)$ and from the bed handles $(n=1)$. The last colony was isolated from the printer of the medical station of the other ICU. The microscopic examination of these colonies revealed A. fumigatus-like conidial head, a large number of cleistothecia, and hyaline, lenticular ascospores with equatorial crests. The $\beta$-tubulin sequences analysis showed a $100 \%$ homology with the reference sequence of $N$. hiratsukae (sequence AF057324).

Neosartorya hiratsukae antifungal susceptibility testing yielded the following MIC values: itraconazole, $0.12 \mathrm{mg} / \mathrm{L}$; voriconazole, $0.03 \mathrm{mg} / \mathrm{L}$; posaconazole, $0.06 \mathrm{mg} / \mathrm{L}$; isavuconazole, $0.12 \mathrm{mg} / \mathrm{L}$; and amphotericin B, $<0.03 \mathrm{mg} / \mathrm{L}$. No break points (BPs) have been specified for N. hiratsukae; however, the MIC values of our isolate are low and under the EUCAST BPs established for A. fumigatus. ${ }^{8}$

No cases of aspergillosis were diagnosed in the 2 ICUs in a 5-month period, 3 before the isolation of $N$. hiratsukae from the environment and 2 thereafter (Dr R. Grande, personal communication). In July 2020, we were able to perform further environmental samplings, but neither $N$. hiratsukae nor other fungi were isolated.

Like all filamentous fungi, Neosartorya also has an environmental origin. In the literature, $N$. hiratsukae has been reported in the indoor air ${ }^{1}$ and on drywall, where the small white colonies are hardly visible, so the spores can easily spread in the environment causing a health risk. ${ }^{9}$ In the 2 ICUs examined, no renovations had 